\title{
Joint Comment on the paper
}

\section{Kinetic Energy and Spatial Width of Ion Clouds in Paul Traps}

\begin{abstract}
M. Desaintfuscien ${ }^{1}$, M. Schubert ${ }^{2}$, I. Siemers ${ }^{2}$, and R. Blatt ${ }^{2}$
Applied Physics B 51, 414 (1990)

In the paper "Kinetic Energy and Spatial Width of Ion Clouds in Paul Traps" by M. Schubert et al. several models describing the dynamics of ion clouds in Paul traps are compared with each other and with experimental results. In this comment we would like to amend the Sect. 3.4 of the paper which is concerned with the work of Meis et al. [1]. In this section there are some errors which we would like to correct:
\end{abstract}

1 Laboratoire de 1'Horloge Atomique, CNRS, Université, Paris-Sud, Bâtiment 221, F-91405 Orsay Cedex, France

2 I. Institut für Experimentalphysik, Universität Hamburg, Jungiusstrasse 9, W-2000 Hamburg 36, Fed. Rep. Germany

(I) Equation (9) should read:

$$
R^{\mathrm{M}}=\frac{1}{\Omega^{2}}\left(\omega_{\mathrm{e}}^{2}+\frac{2}{3} \omega^{2}\right) .
$$

(II) The last expression of Sect. 3.4 should be $R^{\mathrm{M}}(N \rightarrow 0)=\frac{5}{3} \omega^{2} / \Omega^{2}$.

This has to be compared with the result of $R^{\mathrm{mPS}}$ for isotropic pseudopotentials $R^{\mathrm{mPS}}=\frac{3}{2} \omega^{2} / \Omega^{2}$. In view of the approximations inherent in the calculations, these results are in rather good agreement. 